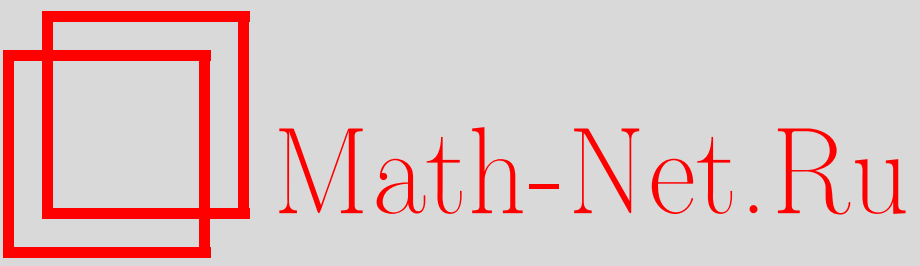

И. М. Арбеков, Критерии секретности ключа, Матем. вопр. криптогр., 2016, том 7, выпуск 1, 39-56

DOI: https://doi.org/10.4213/mvk173

Использование Общероссийского математического портала Math-Net.Ru подразумевает, что вы прочитали и согласны с пользовательским соглашением

http://www . mathnet.ru/rus/agreement

Параметры загрузки:

IP: 3.89 .185 .249

26 апреля 2023 г., 15:51:09 
МАТЕМАТИЧЕСКИЕ ВОПРОСЫ КРИПТОГРАФИИ

2016 T. 7 № 1 C. $39-56$

УДК 519.719.2

\title{
Критерии секретности ключа
}

\section{И. М. Арбеков}

ОАО «ИнфоТеКС», Москва

Получено 8.IX.2015

В работе формализуется понятие практической секретности схемы шифрования, введенное К. Шенноном как «среднего объема работы, необходимой для определения ключа...», и вводится соответствующий критерий практической секретности ключа. Производится сравнение предложенного критерия с энтропийным критерием и критерием расстояния по вариации, используемыми в квантовой криптографии.

Ключевые слова: модели выбора ключей, секретность ключа, оптимизация переборных алгоритмов

\section{Criteria of key security}

\section{M. Arbekov}

InfoTeCS, OJSC, Moscow

\begin{abstract}
We formalize the notion of practical security of the encryption scheme introduced by C.Shannon as "the average amount of work to determine the key..." and define the corresponding practical security of key criterion. A comparison of the proposed criterion with the entropy criterion and the total variance criterion used in the quantum cryptography is given.
\end{abstract}

Keywords: models of key generation, key security, brute force attack optimization

Citation: Mathematical Aspects of Cryptography, 2016, v. 7, № 1, pp. 39-56 (Russian)

(c) Академия криптографии Российской Федерации, 2016 г. 


\section{1. Критерии секретности ключа в квантовой криптографии}

Задачей квантовой криптографии является формирование у легитимных пользователей одинаковой битовой строки $W$, которая может использоваться как ключ в классической криптографической системе и которая является «недоступной» для подслушивателя $[1,2]$.

«Недоступность» понимается в том смысле, что при выполнении определенных требований к квантово-криптографической системе связи подслушивание квантового и открытого каналов не приводит к сильному отличию апостериорного распределения $W$ от априори равновероятного распределения.

В свою очередь, близость апостериорного и априорного распределений оценивается по двум критериям: энтропийному $[3,4]$ и критерию расстояния по вариации [4-9].

Указанные критерии секретности ключа могут быть определены следующим образом.

Имеется ключевое множество $K$ мощности $|K|=N$ с распределением вероятностей $P=\left(p_{1}, p_{2}, \ldots, p_{N}\right)$.

Энтропийный критерий секретности уровня $\varepsilon>0$ определяет близость распределения вероятностей $P=\left(p_{1}, p_{2}, \ldots, p_{N}\right)$ к равновероятному распределению $P_{0}=\left(N^{-1}, N^{-1}, \ldots, N^{-1}\right)$ неравенством

$$
\log _{2} N-H(P) \leqslant \varepsilon
$$

где $H(P)=-\left(p_{1} \log _{2} p_{1}+p_{2} \log _{2} p_{2}+\cdots+p_{N} \log _{2} p_{N}\right)$ есть энтропия по Шеннону распределения вероятностей $P=\left(p_{1}, p_{2}, \ldots, p_{N}\right), \log _{2} N$ - энтропия по Шеннону равновероятного распределения $P_{0}=\left(N^{-1}, N^{-1}, \ldots, N^{-1}\right)$.

Критерий расстояния по вариащзи уровня $\delta>0$ определяет близость распределения вероятностей $P=\left(p_{1}, p_{2}, \ldots, p_{N}\right)$ к равновероятному распределению $P_{0}=\left(N^{-1}, N^{-1}, \ldots, N^{-1}\right)$ неравенством

$$
\frac{1}{2} \sum_{m=1}^{N}\left|p_{m}-\frac{1}{N}\right| \leqslant \delta .
$$

В «новейшей истории» квантовой криптографии (2010 г. и позднее) развернулась острая полемика [10-20] о том, являются ли эти критерии секретности ключа естественными в криптографическом смысле, и в чем именно состоит этот криптографический смысл?

\section{МАТЕМАТИЧЕСКИЕ ВОПРОСЫ КРИПТОГРАФИИ}


Критики предложенных критериев Н. P. Yuen, O. Hirota предлагают взять за основу классическое определение совершенной (теоретической) секретности шифра К. Шеннона [21, с. 361].

Совершенная секретность - это равенство априорных и апостериорных вероятностей:

$$
\mathbf{P}(X)=\mathbf{P}(X \mid Y),
$$

где $X$ - открытое сообщение, $Y$ - соответствующее ему шифрсообщение.

Равенство (1) имеет место при побитовом суммировании по модулю 2 знаков битового сообщения $X$ со знаками случайной равновероятной битовой последовательности.

На основе этого факта в [14] предлагается критерий равномерной (назовем ее так) секретности ключа, который в случае $K=\{0,1\}^{n}$ имеет вид

$$
p_{m} \approx 2^{-n} \quad \text { для всех } \quad m=1,2, \ldots, 2^{n} .
$$

Конкретные отклонения от равновероятности не указываются, ясно, что они должны быть невелики.

В [10] показано, что для случая $K=\{0,1\}^{n}$ и для обоих вышеприведенных критериев - энтропийного и расстояния по вариации - критерий равномерной секретности может не выполняться, поскольку при разумных ограничениях на

$$
\log _{2} N-H(P) \quad \text { и } \quad \frac{1}{2} \sum_{m=1}^{N}\left|p_{m}-\frac{1}{N}\right|
$$

можно указать распределения с вероятностями отдельных ключей, значительно превосходящими $2^{-n}$.

А именно, пусть $p_{1}=\max _{1 \leqslant m \leqslant 2^{n}} p_{m}$. Следуя [10], нетрудно получить, что для распределения $P=\left(p_{1}, p_{2}, \ldots, p_{2^{n}}\right)$, где

$$
\begin{array}{cl}
p_{1}=2^{-d n}, & 0<d<1, \\
p_{m}=\frac{1-2^{-d n}}{2^{n}-1}, & 2 \leqslant m \leqslant 2^{n},
\end{array}
$$

при больших $n$ имеют место следующие соотношения:

$$
\begin{aligned}
\varepsilon_{H} & =\log _{2} N-H(P)=n \frac{1-d}{2^{d n}}\left(1+O\left(\frac{1}{n}\right)\right), \\
\delta_{\rho} & =\frac{1}{2} \sum_{m=1}^{N}\left|p_{m}-\frac{1}{N}\right|=\frac{1}{2^{d n}}\left(1+O\left(\frac{1}{2^{(1-d) n}}\right)\right) .
\end{aligned}
$$

2016, T. 7, № 1, C. 39-56 
Учитывая, что криптографическая защита открытых сообщений определяется сложностью нахождения ключа зашифрования, считаем, что здесь разумно рассмотреть критерий секретности ключа с позиций еще одного положения классической работы К. Шеннона, а именно, положения о практической секретности криптосистемы как «среднего объема работы, необходимой для определения ключа ... » [21, с. 387].

\section{2. Практическая секретность ключа}

\section{1. В чем измерять объем работы до определения ключа}

С самого начала укажем, во-первых, что мы будем понимать под «определением ключа» и, во-вторых, в каких единицах будем измерять «объем работы до определения ключа».

Определение ключа будем связывать с алгоритмами опробования вариантов ключа из некоторого подмножества (может быть, полного) ключевого множества, при этом в обычных для криптографической практики предположениях о наличии шифрсообщения заключительное «определение ключа» (нахождение истинного ключа) будет происходить при читаемости расшифрованного сообщения. Считаем, что критерий истинности ключа (критерий читаемости) у нас есть. Предполагается также, что шифр настолько близок к идеальному, что способы, отличные от опробования, неприменимы.

За единииу объема работы примем опробование одного ключа с проверкой на читаемость сообщения. Тогда если до определения ключа (нахождения истинного ключа) было опробовано $M$ ключей, то полагаем, что объем $R$ работы равен $M$. Ниже будут даны примеры вычисления среднего объема работы.

\section{2. Средний объем работы для алгоритма полного опробования ключей}

Пусть $K$ - ключевое множество с некоторым распределением вероятностей $P=\left(p_{1}, p_{2}, \ldots, p_{N}\right)$, в соответствии с которым ключи выбираются для зашифрования. Будем считать, что ключи упорядочены по вероятностям:

$$
p_{1} \geqslant p_{2} \geqslant \ldots \geqslant p_{N} \text {. }
$$

Очевидно, что наиболее разумным алгоритмом полного опробования ключевого множества является опробование в порядке, задаваемом (3), при этом средний объем работы до определения ключа равен

$$
\bar{R}=\mathbf{E} R=\sum_{m=1}^{N} m p_{m} .
$$


Докажем, что средний объем работы достигает своего максимума на равновероятном распределении $P_{0}=\left(N^{-1}, N^{-1}, \ldots, N^{-1}\right)$ :

$$
\bar{R}^{(\max )}=\sum_{m=1}^{N} m \frac{1}{N}=\frac{N+1}{2} \approx \frac{N}{2} .
$$

Утверждение. Пусть

$$
1>p_{1} \geqslant p_{2} \geqslant \cdots \geqslant p_{N}>0, \quad \sum_{m=1}^{N} p_{m}=1 .
$$

Тогда

$$
\sum_{m=1}^{N} m p_{m} \leqslant \sum_{m=1}^{N} m \frac{1}{N}
$$

Доказательство. Так как $1<2<\cdots<N$ и $p_{1} \geqslant p_{2} \geqslant \cdots \geqslant p_{N}$, то по известному свойству перестановочных сумм (см., например, [22, теорема 368])

$$
\sum_{m=1}^{N} m p_{m} \leqslant \sum_{m=1}^{N} m p_{\sigma_{m}}
$$

для любой перестановки $\sigma=\left(\sigma_{1}, \sigma_{2}, \ldots, \sigma_{N}\right)$ чисел $1,2, \ldots, N$. Суммируя эти неравенства по всем $N$ ! перестановкам $\sigma \in S_{N}$, получаем:

$$
\begin{aligned}
N ! \sum_{m=1}^{N} m p_{m} & \leqslant \sum_{m=1}^{N} m \sum_{\sigma \in S_{N}} p_{\sigma_{m}}=\sum_{m=1}^{N} m \sum_{k=1}^{N} \sum_{\sigma \in S_{N}: \sigma_{m}=k} p_{k}= \\
& =(N-1) ! \sum_{m=1}^{N} m,
\end{aligned}
$$

откуда следует доказываемая оценка.

Теперь мы уже можем сформулировать критерий практической секретности ключа, когда средний объем работы алгоритма опробования близок к максимальному. Но возникает вопрос: а насколько хорош алгоритм полного опробования ключей? Нет ли других алгоритмов, более эффективных по среднему объему работы, необходимой для определения ключа? 


\section{3. Средний объем работы для алгоритма усеченного опробования ключей}

В предыдущем разделе речь шла о ключевом множестве $K$ с упорядоченным распределением вероятностей выбора $P=\left(p_{1}, p_{2}, \ldots, p_{N}\right), p_{1} \geqslant p_{2} \geqslant \cdots$ $\cdots \geqslant p_{N}$ и о среднем объеме работы (математическом ожидании)

$$
\bar{R}=\sum_{m=1}^{N} m p_{m}
$$

до определения истинного ключа.

Согласно В. Феллеру [23, с. 14], говоря о вероятностях, о среднем значении, мы должны представлять себе эксперимент, выполняемый «... очень много раз, подобно бросанию монеты...», где относительные частоты появления ключей, на которых производится зашифрование открытых сообщений, будут близки к их вероятностям $P=\left(p_{1}, p_{2}, \ldots, p_{N}\right)$, а среднее арифметическое значение объемов работы - к математическому ожиданию $\bar{R}$.

Эксперимент по опробованию ключей, повторенный много раз, можно представить как последовательность испытаний Бернулли, где «успехом» считается событие, состоящее в определении истинного ключа (каждый раз нового, выбираемого в соответствии с распределением $\left.P=\left(p_{1}, p_{2}, \ldots, p_{N}\right)\right)$. $\mathrm{B}$ контексте наших рассуждений ясно, что при полном опробовании ключей определение истинного ключа происходит в каждом эксперименте с вероятностью «успеха», равной единице.

Возникает естественный вопрос: как изменится средний объем работы до определения истинного ключа, если применить усеченный алгоритм $U$ и вместо опробования ключей всего упорядоченного ключевого множества $K$ ограничиться только опробованием ключей его начальной части $\widetilde{K} \subset K$ мощности $|\widetilde{K}|=M<N$ с суммарной вероятностью

$$
\pi_{U}(M)=\sum_{m=1}^{M} p_{m}, \quad 0<\pi_{U}(M)<1 ?
$$

В этом случае переменный (как случайная величина) истинный ключ $k_{0}$ определяется не в каждом эксперименте, а с указанной вероятностью «успеха» $\pi_{U}(M)$. Число экспериментов $\xi$ до первого успешного - когда истинный ключ попадает в множество $\widetilde{K}-$ имеет геометрическое распределение

$$
\mathbf{P}\{\xi=r\}=\left(1-\pi_{U}(M)\right)^{r} \pi_{U}(M), \quad r=0,1,2, \ldots,
$$


со средним значением

$$
\mathbf{E} \xi=\frac{1-\pi_{U}(M)}{\pi_{U}(M)} .
$$

Очевидно, что в каждом эксперименте, где выбираемый (истинный) ключ не принадлежит опробуемому множеству, средний объем работы равен

$$
\mathbf{E}\left(R \mid k_{0} \notin \widetilde{K}\right)=M \text {. }
$$

При условии $k_{0} \in \widetilde{K}$ распределение ключей $k_{0}$ имеет вид

$$
\left(\frac{p_{1}}{\pi_{U}(M)}, \frac{p_{2}}{\pi_{U}(M)}, \ldots, \frac{p_{M}}{\pi_{U}(M)}\right),
$$

а средний объем работы при этом условии становится равным

$$
\mathbf{E}\left(R \mid k_{0} \in \widetilde{K}\right)=\sum_{m=1}^{M} m \frac{p_{m}}{\pi_{U}(M)}
$$

Отсюда следует, что средний объем работы до определения ключа для усеченного алгоритма $U$ равен

$$
\begin{aligned}
\bar{R}_{U} & =\bar{R}_{U}(M)=\mathbf{E} \xi \mathbf{E}\left(R \mid k_{0} \notin \widetilde{K}\right)+\mathbf{E}\left(R \mid k_{0} \in \widetilde{K}\right)= \\
& =\frac{1-\pi_{U}(M)}{\pi_{U}(M)} M+\sum_{m=1}^{M} m \frac{p_{m}}{\pi_{U}(M)}= \\
& =\frac{1}{\pi_{U}(M)}\left[\left(1-\pi_{U}(M)\right) M+\pi_{U}(M) \sum_{m=1}^{M} m \frac{p_{m}}{\pi_{U}(M)}\right]= \\
& =\frac{1}{\sum_{m=1}^{M} p_{m}}\left[\left(1-\sum_{m=1}^{M} p_{m}\right) M+\sum_{m=1}^{M} m p_{m}\right]
\end{aligned}
$$

В качестве оценки практической секретности ключа с распределением вероятностей $p_{1} \geqslant p_{2} \geqslant \cdots \geqslant p_{N}$ естественно взять минимальное значение среднего объема работы:

$$
Q=\min _{1 \leqslant M \leqslant N} \bar{R}_{U}(M)
$$

Рассмотрим следующие два простых примера распределений на ключевом множестве (используемых в ходе дискуссии в квантовой криптографии) и изучим поведение величины $Q$. 


\section{Пример 1. Равновероятное распределение}

Пусть $P=P_{0}=\left(N^{-1}, N^{-1}, \ldots, N^{-1}\right), N=2^{n}$. Используя (5), получаем:

$$
\bar{R}_{U}(M)=\frac{\left(1-M / 2^{n}\right) M+2^{-n}(1+M) M / 2}{M / 2^{n}}=\frac{2^{n+1}-M+1}{2} .
$$

Минимальное значение

$$
Q=\min _{1 \leqslant M \leqslant N} \bar{R}_{U}(M)=\frac{2^{n}+1}{2} \approx 2^{n-1}
$$

достигается, очевидно, для $M=2^{n}$, т. е. для алгоритма полного опробования (4).

Таким образом, даже при оптимальном выборе параметра $M$ усеченный алгоритм опробования равновероятного ключа не дает никакого преимущества по сравнению с полным перебором.

\section{Пример 2. Распределение с максимальной первой вероятностью}

Пусть $P=\left(p_{1}, p_{2}, \ldots, p_{N}\right), N=2^{n}, 0<d<1$,

$$
p_{1}=\frac{1}{2^{d n}}, \quad p_{m}=\frac{1-2^{-d n}}{2^{n}-1} \quad \text { при } \quad m=2,3, \ldots, 2^{n} .
$$

Используя (5), получаем:

$$
\begin{aligned}
\bar{R}_{U}(M)= & {\left[\left(1-\frac{1}{2^{d n}}-(M-1) \frac{1-2^{-d n}}{2^{n}-1}\right) M+\frac{1}{2^{d n}}+\frac{1-2^{-d n}}{2^{n}-1} \sum_{m=2}^{M} m\right] \times } \\
& \times\left[\frac{1}{2^{d n}}+(M-1) \frac{1-2^{-d n}}{2^{n}-1}\right]^{-1} .
\end{aligned}
$$

Элементарные выкладки дают асимптотические выражения

$$
\begin{aligned}
\bar{R}_{U}(M) & =\frac{2^{n} M-M^{2} / 2}{2^{n(1-d)}+M}\left(1+O\left(\frac{1}{2^{C^{\prime} n}}\right)\right), \\
\pi_{U}(M) & =\sum_{k=1}^{M} p_{k}=\left(\frac{1}{2^{d n}}+\frac{M}{2^{n}}\right)\left(1+O\left(\frac{1}{2^{C^{\prime \prime} n}}\right)\right)
\end{aligned}
$$

для подходящих постоянных $C^{\prime}, C^{\prime \prime}>0$.

Производная главной части функции $\bar{R}_{U}(M)$ (для упрощения выкладок считаем $M$ непрерывной величиной), равная

$$
\bar{R}_{U}^{\prime}(M)=\frac{2^{n(2-d)}-2^{n(1-d)} M-M^{2} / 2}{\left(2^{n(1-d)}+M\right)^{2}},
$$


имеет единственный положительный корень вида

$$
M^{*}=2^{(n(2-d)+1) / 2}\left(1+O\left(\frac{1}{2^{C^{\prime \prime \prime} n}}\right)\right) .
$$

Нетрудно проверить, что в точках

$$
M_{1}=\frac{1}{2} M^{*}, \quad M_{2}=\frac{3}{2} M^{*}
$$

производная имеет разные знаки: $\bar{R}_{U}^{\prime}\left(M_{1}\right)>0, \bar{R}_{U}^{\prime}\left(M_{2}\right)<0$. Тогда, с учетом единственности положительного корня производной, можно утверждать, что в точке $M^{*}$ имеется максимум главной части функции $\bar{R}_{U}(M)$, а минимум достигается в одной из граничных точек отрезка $1 \leqslant M \leqslant 2^{n}$.

Замечая, что при $d \leqslant(n-1) / n$

$$
\bar{R}_{U}\left(2^{n}\right)-\bar{R}_{U}(1) \approx 2^{n-1}-2^{d n}>0
$$

получаем, что минимум $\bar{R}_{U}(M)$ достигается в левой граничной точке при значении $M=1$. Таким образом,

$$
Q=\min _{1 \leqslant M \leqslant N} \bar{R}_{U}(M)=\bar{R}_{U}(1)
$$

В случае когда $(n-1) / n<d \leqslant 1$ («почти» равновероятное распределение), разность $\bar{R}_{U}\left(2^{n}\right)-\bar{R}_{U}(1) \approx 2^{n-1}-2^{d n}$ отрицательна, поэтому минимум будет справа, как при полном опробовании.

Для иллюстрации результатов положим $n=256, d=0,5$. Тогда минимальное значение среднего объема работы «до определения ключа ... » равно $Q=\bar{R}_{U}(1)=10^{-38,5 \ldots}$, что существенно меньше максимального значения $2^{555}=5,79 \cdot 10^{76}$ для равновероятного случая.

Значение $Q=10^{38,5 \ldots}$ получается при опробовании в каждом эксперименте только одного, первого, ключа, имеющего максимальную вероятность $p_{1}=2^{-128}=10^{38,5 \cdots}$. Нетрудно видеть, что здесь величина $Q$ практически равна $\left(1-p_{1}\right) / p_{1}$ и, по существу, представляет собой среднее число экспериментов (шагов) до появления истинного ключа на первом месте. 
Здесь нельзя оставить без внимания такую величину, как скорость создания шифрсообщений. Действительно, с практической стороны полученная величина $Q=10^{38,5}$ имеет смысл только в том случае, когда криптосистема за весь срок своей «жизни» произведет не менее $10^{38}$ шифрсообщений. Если разумно ограничиться сроком «жизни» в 100 лет, то за это время такой объем должен создаваться со скоростью не менее $10^{28}$ шифрсообщений в секунду. Даже если считать, что мы «следим» за несколькими криптосистемами, необходимая скорость создания шифрсообщений все равно остается запредельно большой. Поэтому при рассмотрении усеченных алгоритмов $U$ со средним объемом работы

$$
\bar{R}_{U}(M)=\frac{1}{\sum_{m=1}^{M} p_{m}}\left[\left(1-\sum_{m=1}^{M} p_{m}\right) M+\sum_{m=1}^{M} m p_{m}\right]
$$

целесообразно ограничиться рассмотрением только таких алгоритмов, для которых

$$
\pi_{U}(M)=\sum_{m=1}^{M} p_{m} \geqslant \pi_{0}
$$

при некотором разумном выборе величины $\pi_{0}$. Напомним, что $\pi_{U}(M)-$ это вероятность успеха или вероятность определения ключа в отдельном эксперименте, при этом среднее число экспериментов (шагов) до появления истинного ключа в перебираемом множестве составит величину, не превосходящую $\pi_{0}^{-1}$.

Здесь следует заметить, что когда мы исключаем из рассмотрения алгоритмы, для которых $\pi_{U}(M)<\pi_{0}$, то как бы допускаем возможность дешифрования (в среднем) доли шифрсообщений не более $\pi_{0}$, т. е. возможность дешифрования (в среднем) каждого $\pi_{0}^{-1}$-го шифрсообщения. Поэтому при выборе $\pi_{0}$ можно также руководствоваться тем, какую часть информации, подлежащей засекречиванию, можно считать доступной для дешифровальщика без особого ущерба для засекречивающей стороны.

Практическую секретность ключа с упорядоченным распределением вероятностей $p_{1} \geqslant p_{2} \geqslant \ldots \geqslant p_{N}$ тогда можно оценить величиной

$$
Q=\min _{M: \pi_{U}(M) \geqslant \pi_{0}} \bar{R}_{U}(M)
$$

Как разумно выбрать $\pi_{0}$ ? Если шифрсообщение создается один раз в сутки, то при $\pi_{0}=10^{-5}$ за 300 лет (примерно) будет прочитано (в среднем!) одно сообщение из $10^{5}$, если один раз в секунду, то при $\pi_{0}=10^{-10}$ за те же 300 лет будет прочитано (в среднем!) одно сообщение из $10^{10}$. 
Положим в примере 2 значение $\pi_{0}$ равным $10^{-10}$ («жесткая» граница вероятности успеха), $n=256, d=0,5$. Решение уравнения $\pi_{U}(M)=\pi_{0}$, отвечающего уравнению (6) вида

$$
2^{-128}+2^{-256} \cdot M=10^{-10},
$$

дает значение $M_{0}=2^{222,8}>M^{*}$, где $M^{*} \approx 2^{(n(2-d)+1) / 2}=2^{192,5}$ есть точка максимума $\bar{R}_{U}(M)$. Тогда, как нетрудно убедиться, для практической секретности справедлива оценка

$$
Q=\min _{M: \pi_{U}(M) \geqslant \pi_{0}} \bar{R}_{U}(M)=\bar{R}_{U}\left(2^{n}\right)=5,79 \ldots \cdot 10^{76} \approx 2^{255} .
$$

Следовательно, для рассматриваемого примера при ограничении $\pi_{0}=10^{-10}$ условие $\pi_{U}(M) \geqslant \pi_{0}$ выполняется только при переборе значительного объема начального множества ключей. При этом ограничении практическая секретность совпадает (с большой точностью) с максимальным средним объемом работы для равновероятного случая.

Таким образом, вероятностная схема распределения ключей, где один ключ имеет гораздо бо́льшую вероятность $\left(2^{-128}\right)$ по сравнению с остальными вероятностями $\left(\approx 2^{-256}\right)$, не отличается от равновероятной схемы с позиций практической секретности ключа с ограничениями на вероятность успеха.

Приведем пример вероятностной схемы распределения ключей, демонстрирующий преимущество усеченных алгоритмов с позиции практической секретности по сравнению с полным опробованием ключевого множества.

\section{Пример 3. Неравновероятная схема испытаний Бернулли}

Пусть двоичные ключи $k=\left(\varepsilon_{1}, \varepsilon_{1}, \ldots, \varepsilon_{n}\right), \varepsilon_{i} \in\{0,1\}$, получены по неравновероятной схеме испытаний Бернулли с повышенной вероятностью нуля: $\mathbf{P}\left\{\varepsilon_{i}=0\right\}=q, \mathbf{P}\left\{\varepsilon_{i}=1\right\}=p, p+q=1, p<q$.

Распределение вероятностей

$$
P=\left(p_{1}, p_{2}, \ldots, p_{N}\right) \quad\left(N=2^{n}, p_{1} \geqslant p_{2} \geqslant \cdots \geqslant p_{N}\right)
$$

этих ключей упорядочено по убыванию в соответствии с возрастанием веса $\nu=\|k\|=\sum_{i=1}^{n} \varepsilon_{i}$ вектора $k=\left(\varepsilon_{1}, \varepsilon_{2}, \ldots, \varepsilon_{n}\right)$ их двоичной записи: максимальная вероятность $p_{1}=q^{n}$ отвечает единственному вектору веса $\nu=0$, далее в (7) располагается множество из $\left(\begin{array}{l}n \\ 1\end{array}\right)$ одинаковых вероятностей, равных $p q^{n-1}$ и отвечающих векторам веса $\nu=1$, и т. д. Очередное множество, отвечающее векторам веса $\nu=r$, содержит в себе $\left(\begin{array}{l}n \\ r\end{array}\right)$ одинаковых вероятностей, равных $p^{r} q^{n-r}$. 
Для простоты изложения и наглядности результатов рассмотрим не все усеченные алгоритмы опробования, а только те, которые включают в опробуемое начальное подмножество ключей все ключевые вектора с весами, не превосходящими заданное целое число из интервала $[0, n]$.

Тогда мощность $M$ опробуемого подмножества равна

$$
M(r)=\sum_{\nu=0}^{r}\left(\begin{array}{l}
n \\
\nu
\end{array}\right)
$$

где $r$ является параметром усеченного алгоритма. Ясно, что при $r=0$ опробуется только один ключ с максимальной вероятностью $p_{1}$, при $0<r<n$ происходит опробование начального ключевого подмножества мощности $M(r)$, случаю $r=n$ соответствует полное опробование ключей.

Рассмотрим средний объем работы

$$
\bar{R}_{U}(M)=\frac{1}{\sum_{m=1}^{M} p_{m}}\left[\left(1-\sum_{m=1}^{M} p_{m}\right) M+\sum_{m=1}^{M} m p_{m}\right]
$$

и вероятность $\pi_{U}(M)=p_{1}+p_{2}+\cdots+p_{M}$.

Зафиксируем $r$. Тогда, как нетрудно видеть,

$$
\pi_{U}(M)=\pi(r)=\sum_{\nu=0}^{r}\left(\begin{array}{l}
n \\
\nu
\end{array}\right) p^{\nu} q^{n-\nu} .
$$

Далее заметим, что первый ключ в подмножестве ключей веса $\nu \geqslant 1$ в упорядоченном распределении (7) имеет номер (нижний индекс), равный

$$
\sum_{j=0}^{\nu-1}\left(\begin{array}{l}
n \\
j
\end{array}\right)+1
$$

последний ключ - номер

$$
\sum_{j=0}^{\nu}\left(\begin{array}{l}
n \\
j
\end{array}\right)
$$

Тогда, воспользовавшись формулой для суммы членов арифметической прогрессии, нетрудно получить, что

$$
\begin{aligned}
\sum_{m=1}^{M} m p_{m} & =\sum_{m=1}^{M(r)} m p_{m}= \\
& =q^{n}+\sum_{\nu=1}^{r} \frac{1}{2}\left(\sum_{j=0}^{\nu-1}\left(\begin{array}{l}
n \\
j
\end{array}\right)+1+\sum_{j=0}^{\nu}\left(\begin{array}{l}
n \\
j
\end{array}\right)\right)\left(\begin{array}{l}
n \\
\nu
\end{array}\right) p^{\nu} q^{n-\nu}
\end{aligned}
$$


и, следовательно,

$$
\begin{aligned}
\bar{R}_{U}(M)= & \bar{R}(r)= \\
= & {\left[\left(1-\sum_{\nu=0}^{r}\left(\begin{array}{l}
n \\
\nu
\end{array}\right) p^{\nu} q^{n-\nu}\right) \sum_{\nu=0}^{r}\left(\begin{array}{l}
n \\
\nu
\end{array}\right)+\right.} \\
& \left.+\left(q^{n}+\sum_{\nu=1}^{r} \frac{1}{2}\left(\sum_{j=0}^{\nu-1}\left(\begin{array}{l}
n \\
j
\end{array}\right)+1+\sum_{j=0}^{\nu}\left(\begin{array}{l}
n \\
j
\end{array}\right)\right)\left(\begin{array}{l}
n \\
\nu
\end{array}\right) p^{\nu} q^{n-\nu}\right)\right] \times \\
& \times\left[\sum_{\nu=0}^{r}\left(\begin{array}{l}
n \\
\nu
\end{array}\right) p^{\nu} q^{n-\nu}\right]^{-1} .
\end{aligned}
$$

Минимальное значение среднего объема работы (при ограничении на вероятность успеха) также будем искать по целочисленному параметру $r$ :

$$
Q=\min _{r: \pi(r) \geqslant \pi_{0}} \bar{R}(r)
$$

Возьмем для численных расчетов значения $n=256, p=0,4, q=0,6$ и построим график функции $L(r)=\log _{2} \bar{R}(r)$ в целочисленных точках $0 \leqslant r \leqslant n$.

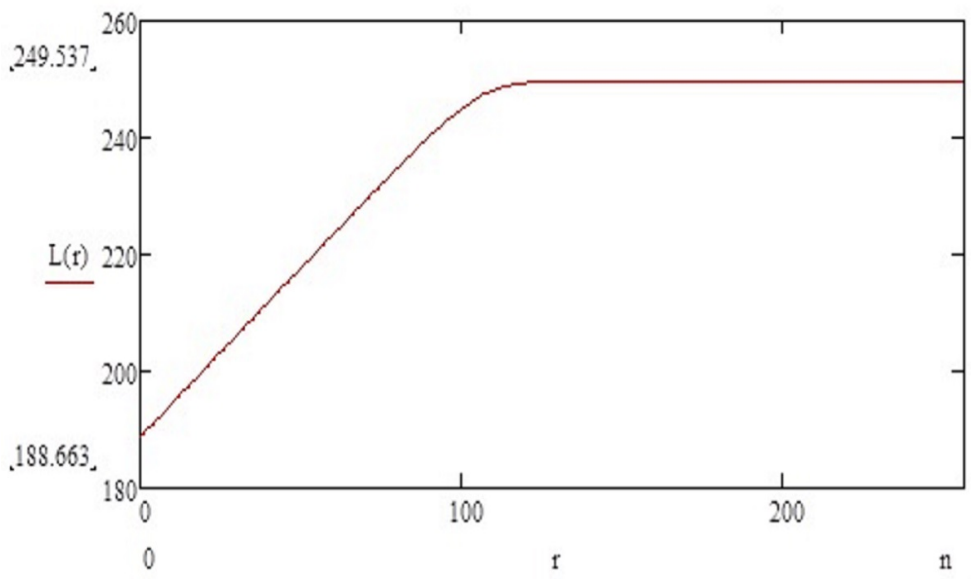

Рис. График функции $L(r)$ при $n=256, \quad p=0,4, \quad q=0,6$

Из графика видно, что значения $L(r)=\log _{2} \bar{R}(r)$ не убывают при возрастании целочисленного аргумента $r$. 
Учитывая также, что

$$
\pi(r)=\sum_{\nu=0}^{r}\left(\begin{array}{l}
n \\
\nu
\end{array}\right) p^{\nu} q^{n-\nu}
$$

возрастает с ростом $r$, для целочисленного аргумента имеем:

$$
Q=\min _{r: \pi(r) \geqslant \pi_{0}} \bar{R}(r) \leqslant \bar{R}\left(r_{0}\right)
$$

где $r_{0}=\min \left\{r: \pi(r) \geqslant \pi_{0}\right\}$.

Положим $\pi_{0}=10^{-10}$ («жесткая» граница вероятности успеха). Вычисляем $r_{0}=55, Q \leqslant \bar{R}\left(r_{0}\right)=2^{220,5 \cdots}$.

Для $\pi_{0}=10^{-5}$ («мягкая» граница вероятности успеха) получаем $r_{0}=70$, $Q \leqslant \bar{R}\left(r_{0}\right)=2^{229,1 \ldots}$.

В случае полного опробования (при $\pi_{0}=1$ ) получаем $r_{0}=n, Q=\bar{R}(n)=$ $=2^{249,5 \ldots}$.

Таким образом, в рассмотренном примере наблюдается заметное снижение среднего объема работы $Q$ (практической секретности ключа) для усеченных алгоритмов по сравнению с полным опробованием ключевого множества.

Теперь мы готовы сформулировать критерий практической секретности ключа, когда средний объем работы алгоритма опробования близок к максимальному среднему объему.

\section{3. Критерий практической секретности ключа}

Определение 1. Случайный ключ с упорядоченным распределением вероятностей $P=\left(p_{1}, p_{2}, \ldots, p_{N}\right)\left(p_{1} \geqslant p_{2} \geqslant \cdots \geqslant p_{N}\right)$ называется $\left(\pi_{0}, \lambda\right)$-практически секретным, если

$$
\log _{2} \frac{N}{2}-\log _{2} Q \leqslant \lambda
$$

где

$$
\begin{aligned}
Q & =\min _{M: \pi_{U}(M) \geqslant \pi_{0}} \bar{R}_{U}(M) \\
\bar{R}_{U}(M) & =\frac{1}{\pi_{U}(M)}\left[\left(1-\pi_{U}(M)\right) M+\sum_{m=1}^{M} m p_{m}\right]
\end{aligned}
$$

- минимальный средний и средний объемы работы до определения истинного ключа для усеченного алгоритма $U$, состоящего в опробовании начальной части ключевого множества мощности $M, \pi_{U}(M)=p_{1}+p_{2}+\cdots+p_{M}$ есть вероятность попадания истинного ключа в опробуемое множество. 
Величина $\log _{2}(N / 2)-\log _{2} Q$ показывает, на сколько двоичных порядков средний объем работы алгоритма опробования неравновероятного ключа меньше максимального среднего объема работы для равновероятного случая, и в таком качестве служит критерием практической секретности ключа.

Ниже проведем сравнение критериев секретности ключа (п. 1 настоящей статьи) для примера 2 - распределения с максимальной первой вероятностью. Нас будет интересовать вопрос о том, при каких значениях $\varepsilon$ энтропийного критерия и $\delta$ критерия расстояния по вариации будет выполняться критерий (8) практической секретности.

Итак, пусть имеется распределение $P=\left(p_{1}, p_{2}, \ldots, p_{N}\right), N=2^{n}$,

$$
\begin{array}{cl}
p_{1}=2^{-d n}, & 0<d<1, \\
p_{m}=\frac{1-2^{-d n}}{2^{n}-1}, & 2 \leqslant m \leqslant 2^{n} .
\end{array}
$$

Положим для численных расчетов (аналогичных приведенным в п. 2.3) $n=256$. Примем также допущение $\lambda=10$, обеспечивающее уменьшение (в двоичных порядках) максимального среднего объема работы от $2^{255}$ до $2^{245}$.

Тогда при $\pi_{0}=10^{-5}$ («мягкая» граница вероятности успеха) неравенство

$$
\log _{2} 2^{255}-\log _{2} Q<10
$$

выполняется при значениях $d>0,065 \ldots$ (чем больше $d$, тем мы ближе к равновероятному распределению) и, следовательно, здесь

$$
\begin{gathered}
\varepsilon_{H}=\log _{2} N-H(P) \approx n \frac{1-d}{2^{d n}}<2,5 \cdot 10^{-3}, \\
\delta_{\rho}=\frac{1}{2} \sum_{m=1}^{N}\left|p_{m}-\frac{1}{N}\right| \approx \frac{1}{2^{d n}}<9,8 \cdot 10^{-6} .
\end{gathered}
$$

При $\pi_{0}=10^{-10}$ («жесткая» граница вероятности успеха) неравенство

$$
\log _{2} 2^{255}-\log _{2} Q<10
$$

выполняется при значениях $d>0,130 \ldots$, и, следовательно, при

$$
\begin{gathered}
\varepsilon_{H}=\log _{2} N-H(P)<2,14 \cdot 10^{-8}, \\
\delta_{\rho}=\frac{1}{2} \sum_{m=1}^{N}\left|p_{m}-\frac{1}{N}\right|<9,6 \cdot 10^{-11} .
\end{gathered}
$$


Эти отклонения достаточно приемлемы и при выполнении соответствующих неравенств (10), (11) гарантируется (для рассматриваемого примера с максимальной первой вероятностью ключа) выполнение критерия практической секретности (9).

В этой связи представляется, что критерий равномерной секретности (2), предложенный в [10], является слишком «жестким» для обеспечения условий практической невозможности определения ключа.

\section{4. Критерий практической секретности (стойкости) схемы шифрования}

Предложенный в настоящей работе критерий практической секретности ключа может быть легко обобщен на критерий практической секретности или стойкости схемы шифрования относительно широкого класса переборных алгоритмов, определяющих ключ с некоторой вероятностью.

Действительно, в числителе выражения (5) стоит средний объем работы (выраженный в числе опробуемых вариантов ключа) алгоритма, не обязательно приводящего к определению ключа, в знаменателе - вероятность определения ключа.

Предположим теперь, что в каждом эксперименте, при каждом применении криптографического алгоритма $A$ определение ключа происходит или не происходит при опробовании некоторого множества ключей $\widetilde{K}$, быть может, случайного. Вероятность успешного применения алгоритма - вероятность попадания истинного ключа $k_{0}$ в переборное множество $\widetilde{K}-$ равна $\pi_{A}$. Тогда

$$
\bar{S}_{A}=\left(1-\pi_{A}\right) \mathbf{E}\left(R \mid k_{0} \notin \widetilde{K}\right)+\pi_{A} \mathbf{E}\left(R \mid k_{0} \in \widetilde{K}\right)
$$

есть средний объем работы алгоритма $A$ (среднее число опробуемых ключей $R$ ) на один эксперимент,

$$
\bar{R}_{A}=\frac{\bar{S}_{A}}{\pi_{A}}=\frac{1-\pi_{A}}{\pi_{A}} \mathbf{E}\left(R \mid k_{0} \notin \widetilde{K}\right)+\mathbf{E}\left(R \mid k_{0} \in \widetilde{K}\right)
$$

есть средний объем работы (среднее число опробуемых ключей) алгоритма $A$ в последовательных экспериментах до определения ключа (нахождения истинного ключа), а

$$
Q=\min _{A: \pi_{A} \geqslant \pi_{0}} \bar{R}_{A}
$$

- оценка практической секретности (стойкости) схемы шифрования. Минимум берется по всем допустимым алгоритмам $A$, для которых вероятность успеха не меньше $\pi_{0}$. 
Определение 2. Схема шифрования, использующая $N$ ключей, называется $\left(\pi_{0}, \lambda\right)$-практически секретной (стойкой), если

$$
\log _{2} \frac{N}{2}-\log _{2} Q \leqslant \lambda,
$$

где величина $Q$ определяется выражением (12).

Значение разности в левой части последнего выражения и кладется в основу критерия практической секретности (стойкости) схемы шифрования.

В заключение дадим еще одну полезную трактовку величины $\bar{R}_{A}$. Если предположить, что при каждом использовании ключа зашифровывается одно сообщение, то при атаке на $L$ сообщений средняя проделанная работа составит $L \bar{S}_{A}$, при этом будет дешифровано $L \pi_{A}$ сообщений. Средний объем работы, затраченный на дешифрование одного сообщения, очевидно, равен

$$
\frac{L \bar{S}_{A}}{L \pi_{A}}=\bar{R}_{A} .
$$

Таким образом, в этом контексте $\bar{R}_{A}$ - это средний объем работы (среднее количество опробуемых ключей), затраченной на дешифрование одного сообщения алгоритмом $A$. Следует отметить, что именно в этом смысле в [24] вводится (без необходимой математической строгости и деталировки) понятие величины, аналогичной $\bar{R}_{A}$.

\section{Список литературы}

[1] Bennett C. H., Brassard G., "Quantum cryptography: public key distribution and coin tossing". In: "Proceedings of IEEE International Conference on Computers, Systems and Signal Processing", Bangalore, India, December 9-12, 1984, Bangalore: Indian Inst. Sci., 1984, 175179.

[2] Inarmori H., Lütkenhaus N., Mayers D., "Unconditional security of practical quantum key distribution”, Eur. Phys. J., Ser. D, 41 :33 (2007), 599-627.

[3] Тимофеев А. В., Помозов Д. И., Маккавеев А. П., Молотков С. Н., “О структуре открытого классического канала связи в квантовой криптографии: коррекция ошибок, целостность и аутентичность”, ЖЭТФ, $131: 5$ (2007), 771-789.

[4] Bennett C.H., Brassard G., Crepeau C., Maurer U. M., "Generalized privacy amplification", IEEE Trans. Inform. Theory, IT-41 :6, Part 2 (1995), 1915-1923.

[5] Tomamichel M., Lim C.C.W., Gizin N., Renner R., "Tight finite-key analysis for quantum cryptography”, Nature Comm., 3 :634 (December 2012), 6, doi: 10.1038/ncomms1631.

[6] Dodis Y., Ostrovsky R., Reyzin L., Smith A., "Fuzzy extractors: how to generate strong keys from biometrics and other noisy data", SIAM J. Comput., 38:1 (2008), 97-139.

[7] Scarani V., Renner R., "Security bounds for quantum cryptography with finite resourses" (2008), http://arxiv.org/abs/0806.0120. 
[8] Chistandl M., Renner R., Ekert A., “A generic security proof for quantum key distribution” (2004), http://arxiv.org/abs/quant-ph/0402131.

[9] Renes J.M., Renner R., "Noisy channel coding via privacy amplification and information reconciliation" (2012), http://arxiv.org/abs/1012.4814.

[10] Yuen H.P., "Fundamental quantitative security in quantum key generation" (2010), http:// arxiv.org/pdf/1008.0623v2.pdf.

[11] Yuen H. P., "Problems of exiting unconditional security proofs in quantum key distribution" (2011), http://arxiv.org/abs/1109.1051.

[12] Yuen H. P., "Fundamental and practical problems of QKD security - the actual and the perceived situation" (2012), http://arxiv.org/abs/1109.1066.

[13] Yuen H.P., "Unconditional security in quantum key distribution" (2012), http://arxiv.org/ abs/1205.5065.

[14] Yuen H. P., "Incompleteness and limit of quantum key distribution theory" (2012), http:// arxiv.org/abs/1208.2106.

[15] Renner R., "Reply to recent scepticism about the foundations of quantum cryptography" (2012), http://arxiv.org/abs/1209.2423.

[16] Yuen H.P., "On the foundations of quantum key distribution - reply to Renner and beyond" (2012), http://arxiv.org/abs/1210.2804.

[17] Hirota O., "Misconception in theory of quantum key distribution - reply to Renner" (2013), http://arxiv.org/abs/1306.1277.

[18] Yuen H.P., "Essential lack of security in quantum key distribution" (2013), http://arxiv.org/ abs/1310.0842.

[19] Yuen H. P., "Can quantum key distribution be secure" (2014), http://arxiv.org/abs/1405.0457.

[20] Hirota O., "A correct security evaluation of quantum key distribution" (2014), http://arxiv.org/ abs/1409.5991.

[21] Шеннон К., Работы по теории информации и кибернетике, М.: ИЛ, 1963, 832 с.

[22] Харди Г. Г., Литтльвуд Дж. Е., Полиа Г., Неравенства, /Пер. с англ. В. И. Левина, М. : ИЛ, 1948, $456 \mathrm{c}$.

[23] Феллер В., Введение в теорию вероятностей, /пер. с англ. Ю.В.Прохорова, М.: Мир, 1984, $528 \mathrm{c}$.

[24] Фомичев В. М., Дискретная математика и криптология, /под общ. ред. Н. Д. Подуфалова, М. : Диалог-МИФИ, 2003, 400 с. 